\title{
Effects of Breaking up Deskwork with Physical Activity Combined with Tea Consumption on Cerebrovascular Function, Mood, and Affect
}

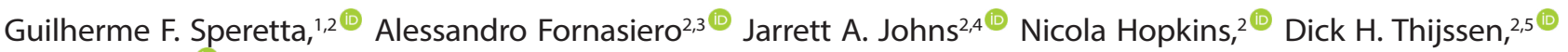 \\ David A. Low ${ }^{\circledR}$ \\ Universidade Federal de Santa Catarina, ${ }^{1}$ Florianópolis, SC - Brazil \\ Liverpool John Moores University, ${ }^{2}$ Liverpool - United Kingdom \\ University of Verona, ${ }^{3}$ Verona - Italy \\ Dalhousie University Halifax, ${ }^{4}$ Nova Scotia - Canadá \\ Radboud University Medical Center, ${ }^{5}$ Nijmegen - Netherlands
}

\section{Abstract}

Background: Prolonged sitting, typical of desk work, decreases cerebral blood flow (CBF), mood and affect. Conversely, short physical activity breaks from sitting may prevent these detrimental effects and provide cardiometabolic benefits.

Objective: We evaluated the effect of interrupting prolonged sitting with short breaks of light physical activity combined with tea consumption on CBF, cerebral autoregulation (CA), mood, and affect in desk workers.

Methods: Nineteen healthy desk workers (ten male, $27 \pm 10$ years) performed desk work in a laboratory for six hours on two separate intervention days: tea breaks (TEA-BREAK: short walk combined with ingestion of one cup of tea every hour) and sedentary (SED: ingestion of one cup of water every hour, while seated). Before and after desk work, we assessed mean arterial pressure (MAP), middle cerebral artery blood velocity (MCAv) and CA. Questionnaires were used to assess mood (Bond \& Lader, PANAS) and affect (Affect grid) before and after the intervention. Data are expressed as mean \pm standard deviation. Two-way ANOVA with repeated measurements followed by Sidak post hoc test was used for data analysis. Paired Student's t-test was also used to compare changes $(\Delta)$ between trials. Statistical significance was at $\mathrm{p}<0.05$.

Results: Desk work increased MAP (4.6 $\pm 4.6 \Delta \mathrm{mmHg} ; \mathrm{P}<0.05)$, and decreased MCAv $(-5.2 \pm 7.0 \Delta \mathrm{cm} / \mathrm{s} ; \mathrm{P}<0.05)$, with no difference between interventions in these parameters. TEA-BREAKS, but not SED, decreased gain $\left(-0.08 \pm 0.12 \Delta \mathrm{cm} . \mathrm{s}^{-1}\right.$. mmHg. $\left.{ }^{-1}\right)$ and increased phase $(5.26 \pm 8.84 \Delta$ radians) at very low frequency $(\mathrm{P}<0.05)$, but not at low frequency. Small changes in positive affect were found after the six hours of desk work $(-5.5 \pm 7.3 \Delta$ scale; $\mathrm{P}<0.05)$, with no differences between interventions.

Conclusion: Changes in MCAv and positive affect induced by prolonged desk work could not be prevented by TEABREAKS. However, TEA-BREAKS improved CA, suggesting a higher efficiency in maintaining MCAv in response to blood pressure fluctuations.

Keywords: Tea; Black Tea; Blood Pressure; Risk Factors; Homeostasis Cerebral; Cerebrovascular Circulation.

\section{Introduction}

Modern lifestyle is marked with unhealthy habits, including a sedentary behavior characterized by several hours of low energy expenditure, such as prolonged uninterrupted sitting. ${ }^{1}$ Sedentary lifestyle is a worldwide public health problem, ${ }^{2}$ primarily due to its association with increased risk of chronic diseases, such as type 2 diabetes, cancer, osteoporosis and cardiovascular disease (CVD) ${ }^{3-5}$ In addition, prolonged sitting decreases cerebral blood flow $(\mathrm{CBF})$ and function, ${ }^{6}$ and may also impair affective and cognitive measures related to mood, affect, and alertness. $^{7}$ 
Public health recommendations suggest reducing sedentary time and increasing physical activity whenever possible. ${ }^{2}$ It has been demonstrated that frequent shortduration walking breaks (mor than walking breaks), can prevent $\mathrm{CBF}$ decreases, promote metabolic benefits (e.g. insulin sensitivity), and enhance mood. ${ }^{6-8}$ However, one potential limitation of these studies is the fact that they used controlled physical activity interventions, which may not be applied to a real-life situation. Usually, in real time situations, physical activity breaks are short and with a very low intensity (i.e., standing), and are often combined with consumption of a beverage.

Epidemiological studies have suggested that regular consumption of tea, one of the most consumed beverages in the world, may improve cardiovascular health and affective function. ${ }^{9,10}$ In fact, recent studies have confirmed that the consumption of black tea is an effective approach to improve cardiovascular function. ${ }^{11,12}$ Furthermore, daily consumption of tea seems to reduce the risk for heart disease and ischemic stroke. ${ }^{13}$ Tea consumption has also been linked to benefits related to attention, alertness, mood and creativity. ${ }^{9}$

Although taking a break for a hot drink is a typical behavior among office-based workers, there is no study examining the effect of this behavior on cerebrovascular and affective function in a research setting. Therefore, this exploratory study evaluated the effects of light, short physical activity breaks with tea consumption at regular intervals during prolonged sitting in healthy, young office workers. We hypothesized that regular breaks associated with tea consumption would prevent prolonged sitting-induced decreases in $\mathrm{CBF}$, and improve cerebral autoregulation (CA), mood and affect.

\section{Methods}

\section{Participants}

Nineteen sedentary desk workers (10 males) aged 20-55 years old were recruited. Participants were screened for the following exclusion criteria: physical exercise $\geq 4$ hours per week, participation in night shift work two weeks prior to screening or during the study, use of dietary supplements, use of prescribed or over-the-counter medication, smoking and history of CVD. The sample size calculation was based on a residual standard deviation of $12 \mathrm{~cm} \cdot \mathrm{s}^{-1}$ in CBF and a correlation of 0.7 between pre- and post-treatment scores reported in a previous study. ${ }^{6}$ With 19 participants in this cross over design, we anticipated a priori that our sample size would be sufficient to detect a difference in middle cerebral artery blood velocity (MCAv) of $10 \%$ between the uninterrupted sitting and the tea break intervention, assuming a power of 0.80 and significance level of 0.05 (2-sided). Study procedures were approved by the Ethics Committee of Liverpool John Moores University (19/SPS/023). Fully informed written and verbal consent was obtained from all participants. Each participant received $£ 50$ for their participation in the study. The study has been registered as a clinical trial in ClinicalTrials.gov (NCT03953391).

\section{Design and experimental procedures}

Participants attended the laboratory at the same time of day (7:00 -8:00 A.M.) on two separate occasions (two interventions). The trials were separated by a 2 to 7 day wash-out, and participants were randomly allocated to one of the interventions: 1 . tea break intervention (TEABREAKS), where, during the sedentary work (desk work, for 6 hours), participants were instructed to have regular breaks ( 5 minutes every hour) from sitting desk work, walk a short distance and prepare and consume tea; or 2. sedentary intervention (SED), where participants were offered water every hour while seated in upright posture at their desk. The randomization sequence was created using Excel 2016 (Microsoft, Redmond, WA, USA) with a 1:1 allocation using random block to ensure a balanced distribution between the two intervention. The hourly breaks were chosen based on previous work, ${ }^{6}$ and on the real-world situation. The duration of breaks (5 minutes) in TEA-BREAKS was determined based on the pilot study conducted in our laboratory to evaluate the feasibility of the study. Testing procedures were the same on all test days. As participants arrived at the laboratory, body mass and height were measured. Then, after 20 minutes of supine rest, MCAv, blood pressure (BP), and heart rate (HR) were assessed, and subsequently participants underwent measurements of CA. After baseline measurements, three questionnaires assessing mood and affect were administered. Then, participants performed either the TEA-BREAKS or the SED. At the end of the six hours of desk work, the questionnaires, and MCAv and CA measurements were repeated (Figure 1).

\section{Interventions}

SED: $150 \mathrm{~mL}$ of tap water at room temperature were provided by the researcher once every hour (total of 5) to participants while sitting at their desks. 


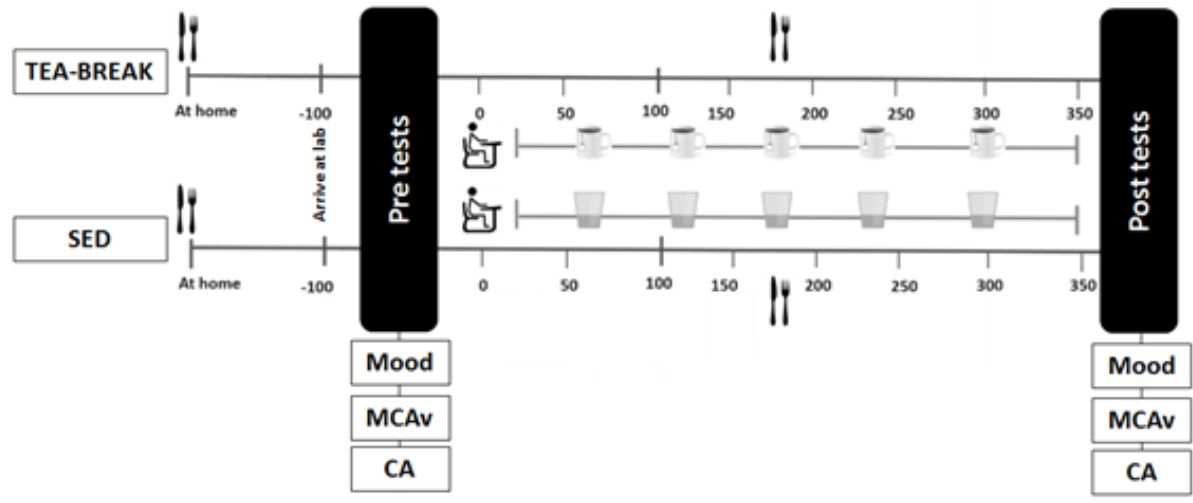

Figure 1 - Experimental design. TEA-BREAK, $6 \mathrm{~h}$ of deskwork with tea breaks every hour. SED, $6 \mathrm{~h}$ of deskwork sedentary intervention drinking water every hour. MOOD, mood questionnaires. MCAv, medium cerebral artery velocity. CA, cerebral autoregulation. Knife and fork represent breakfast and lunch.

TEA-BREAK: Participants were instructed to leave their desk for approximately 5 minutes once every hour. During this period, the subjects were instructed to walk 150 meters at their preferred walking speed to the tea brewing area. Participants were instructed to prepare a black tea (PG Tips pyramid bags, Unilever UK Ltd), by putting the teabag into $150 \mathrm{~mL}$ of boiling water and leaving it to steep for three minutes without stirring. Adding milk and/or sugar was not permitted. Participants then returned to the desk and drank the tea.

Participants were asked to refrain from vigorous exercise for at least 24 hours prior to the sessions and to avoid dietary products that may affect endothelial function, such as caffeine, alcohol, chocolate, and vitamin C for at least 18 h. ${ }^{14,15}$ A standardized breakfast was provided to all participants to be consumed at home one hour prior to laboratory visit, and on both study days, all participants received lunch at the end of the third hour of intervention. Both meals were composed of a medium banana and porridge (Oat So Simple 57g, Quaker, Leicester, UK). For lunch, participants walked to a next-door room to eat their lunch sitting at a table within a 30-minute period. Participants were allowed to move their legs and feet while sitting, to mimic a real-world situation and to prevent the decline in leg blood flow and reduce the risk of thrombus formation. ${ }^{16}$ Participants were instructed to perform only low cognitively demanding desk-based activities, such as reading or completing simple work tasks on a computer. Demanding tasks such as gaming and taking phone calls were not permitted. Participants performed comparable deskbased activities on both test days. Participants were supervised at all times and asked to avoid standing or walking. All participants were asked to visit the toilet before the start of the intervention and at the lunch break. If needed, more toilet breaks were allowed, and participants were taken to the restroom on a wheelchair. Women were evaluated in the early follicular phase of the menstrual cycle to control for hormonal fluctuations.

\section{Measurements}

All measurements were continuously acquired using an analogue-to-digital converter (PowerLab ML880; ADInstruments, Colorado Springs, CO, USA) and displayed in real time on a computer with commercially available software (LabChart version 7.0; ADInstruments).

\section{Blood pressure}

Arterial blood pressure (BP) was measured with an automated sphygmomanometer (Dinamap Procare 100 GE Medical Systems Ltd., Buckinghamshire, UK) ${ }^{17}$ on the left arm while the participant was lying supine. This measure was used to determine resting BP and to calibrate the beat-to-beat BP, which was measured by finger photoplethysmography (Finometer, Finapres Measurement Systems, Arnhem, Netherlands). A cuff was attached to the second phalanx of the right index or middle finger placed at heart level. ${ }^{18}$ 


\section{Middle cerebral artery blood flow velocity}

Continuous bilateral transcranial Doppler ultrasound (TCD) (ST3; Spencer Technologies, Redmond, WA) was used to measure the left and right MCAv. A 2-MHz Doppler probe was positioned over the temporal window, located above the zygomatic arch, and was secured using an adjustable headband (Marc 600 Headframe; Spencer Technologies). Each MCA was identified based on the signal depth, peak, and mean blood flow velocity as previously described. ${ }^{19}$ Once optimal signals had been obtained, the transducers were secured in position, and the signal parameters were recorded to ensure within-subject consistency between tests. Mean MCAv was calculated from the envelope of the velocity tracing using a weighted mean (1/3 maximum 2/3 minimum) to account for the relative time spent in systolic and diastolic pressures..$^{20}$ Supine $\mathrm{MCAv}$ was acquired for 5 minutes before and at the end of the intervention. Cerebral conductance index $\left(\mathrm{CVC}_{\mathrm{i}}\right)$ was calculated by dividing MCAv by mean arterial pressure (MAP). Partial pressure of end-tidal $\mathrm{CO}_{2}\left(\mathrm{PETCO}_{2}\right)$ was sampled with a mouthpiece and monitored via an online gas analyzer (ML206; ADInstruments, Colorado Springs, CO).

\section{Cerebral autoregulation}

Participants completed two repeated cycles of squatting and standing to induce oscillations in BP. Sets were performed at low frequency $(\mathrm{LF} ; 0.1 \mathrm{~Hz} ; 5$ seconds of squatting, followed by 5 seconds of standing) and very low frequency (VLF; $0.05 \mathrm{~Hz} ; 10$ seconds of squatting, followed by 10 seconds of standing), for 5 minutes each, with a 5-minute rest interval. ${ }^{21} \mathrm{MCAv}$ and BP were continuously assessed.

Data were analyzed using transfer function analysis (TFA) as previously described. ${ }^{6,21}$ Briefly, beat-to-beat MCAv and BP signals were spline interpolated and resampled at $4 \mathrm{~Hz}$ for spectral and TFA based on the Welch algorithm. The five-minute data were subdivided into five successive windows that overlapped 50\%. Data contained in each window were linearly detrended and passed through a Hanning window. Then, the discrete Fourier transform was applied. The cross-spectrum between MCAv and BP was determined and divided by the autospectrum of mean arterial pressure (MAP) to derive the TFA coherence, gain, and phase. ${ }^{6,21}$ Phase shift is considered an alternative measure for the time delay of the autoregulatory reactivity, with rises in phase indicating a more efficient CA. Gain describes how changes in BP are transmitted into $\mathrm{MCAv}$, with lower gain indicating a higher CA sensitivity. Coherence labels the linearity of the relationship between the fluctuations in MCAv and BP, with a coherence value approaching one indicating a linear relationship. ${ }^{22,23}$ Coherence values were used to accept the validity of gain and phase estimates, with cut-off values for inclusion set at $0.5 .^{23}$ TFA is a frequency-dependent phenomenon and, as a result, the frequency ranges have different responses and are likely controlled by different mechanisms. The regulation of CBF is efficient in the LF range of $\mathrm{BP}$ oscillations but not in the high frequency range due to the time delay in initiating cerebrovascular adaptations to the changes in perfusion pressure. CA therefore allows rapid $\mathrm{BP}$ changes to be transmitted to $\mathrm{CBF}$, whereas slow $\mathrm{BP}$ changes are filtered. ${ }^{22,23}$ Data were processed and analyzed in agreement with standardized TFA guidelines for the two frequency domains: VLF (0.02$0.07 \mathrm{~Hz})$ and LF $(0.07-0.2 \mathrm{~Hz}){ }^{23}$

\section{Mood and affect}

Three different questionnaires were used to evaluate mood and affect 1. Positive and Negative Affect Schedule (PANAS): consists of a list of ten positive and ten negative feelings and emotions (e.g., active, determined, afraid, irritable). Participants were instructed to rate the extent to which they were currently feeling each emotion on a scale of 1 (very slightly or not at all) to 5 (extremely); ${ }^{24} 2$. Bond-Lader Visual Analogue Scale: this scale comprises of 16 items consisting of an adjective pair (e.g. tense/ relaxed) and a $100 \mathrm{~mm}$ line. Participants were asked to mark on line to what extent the described state was appropriate to them at that moment. ${ }^{25} 3$. The Affect grid: this is a single-item measure of the two affect dimensions of pleasure and arousal, designed as a 10x10 grid. The horizontal line represents the degree of pleasure (ranging from unpleasant to very pleasant), while the vertical line represents the degree of arousal (ranging from sleepy to very active) ${ }^{26}$ Participants were asked to check a cell in the 10x10 grid that best represented how they were feeling in relation to the two dimensions at the moment.

\section{Statistical analysis}

Data are expressed as mean \pm standard deviation (SD) and were analysed using statistical software (Graph Pad Prism 6.0, San Diego, CA, USA). Data were initially tested for normality using the Kolmogorov-Smirnov test. Twoway ANOVA with repeated measurements followed by Sidak post hoc test was used to analyse all variables. Paired Student's t-test was also used to compare changes 
$(\Delta)$ between interventions. Statistical significance was set at $\mathrm{p}<0.05$.

\section{Results}

\section{Descriptive statistics are presented in Table 1.}

\section{Baseline cardiorespiratory and hemodynamic parameters}

The two-way ANOVA analysis showed a significant time effect for systolic arterial pressure (SAP), diastolic arterial pressure (DAP), MAP, MCAv, $\mathrm{CVC}_{\mathrm{i}}$, and $\mathrm{HR}$. We found increases in SAP, DAP and MAP and decreases in $\mathrm{MCAv}, \mathrm{CVC}_{\mathrm{i}}$, and HR after 6 hours of sitting. However, no effect of the intervention was observed on any of these parameters. Analysis of peak MCAv and MCAv corrected for baseline values also showed a decrease in these parameters over time with no significant difference between the interventions (data not shown). Also, comparisons of MCAv were made between men and women, and no significant differences were observed (data not shown). There was also a significant time effect, and an interaction effect for $\mathrm{PETCO}_{2}$, but no effect of the intervention (Table 2). Sidak's post-hoc test revealed decreases in $\mathrm{PETCO}_{2}$ in the TEA-BREAK intervention. Student's t-test analysis showed a greater decline in relative $\mathrm{PETCO}_{2}$ after the TEA-BREAK intervention compared to SED.

\section{Cerebral autoregulation}

The two-way ANOVA analysis showed a time effect in the VLF for gain but not for phase, and no effect (either for gain or phase) of intervention. An interaction effect was observed in VLF for both phase and gain, with post hoc analysis showing decreases in gain after the TEABREAK trial, but not after SED. Student's t-test analysis showed decreases in the relative gain and increases in phase in the TEA-BREAK intervention compared to SED intervention in the VLF (Figure 2). In LF, no effect of time, intervention or interaction effects on gain or phase was detected by the two-way ANOVA or the Student's t-test (Figure 3). Mean coherence values were 0.8 in the VLF and 0.6 in the LF (pre- and post-intervention).

\section{Mood and affect questionnaires}

The two-way ANOVA showed a time effect in The PANAS questionnaire for positive affect, with Sidak's post hoc analysis showing decreases in affect from pre- to post-SED intervention. Student's t-test analysis showed no differences between TEA-BREAK and SED for affect. No other significant effects were observed (Table 3 ).

\section{Discussion}

The purpose of this study was to assess the effects of sitting breaks combined with tea consumption during prolonged sitting (every hour for six hours), reflective of a sedentary desk work, on CBF and function, affect, and mood, in healthy young workers. We found that prolonged desk work decreased CBF (measured by MCAv) and positive affect, but regular breaks with physical activity (short walk) and tea consumption were not able to prevent these changes. However, work sitting breaks with a short walk and tea consumption increased phase and reduced gain in CA, reflecting a quicker and

Table 1 - Characteristics of participants $(n=19)$

\begin{tabular}{ll}
\hline & Mean \pm SD \\
\hline Age (years) & $27 \pm 10$ \\
\hline Body mass $(\mathrm{kg})$ & $70 \pm 11$ \\
\hline Height $(\mathrm{m})$ & $1.7 \pm 0.1$ \\
\hline Body mass index $\left(\mathrm{kg} / \mathrm{m}^{2}\right)$ & $23 \pm 3$ \\
\hline Systolic arterial pressure $(\mathrm{mmHg})$ & $114 \pm 7$ \\
\hline Diastolic arterial pressure $(\mathrm{mmHg})$ & $65 \pm 6$ \\
\hline Mean arterial pressure $(\mathrm{mmHg})$ & $81 \pm 5$ \\
\hline Heart rate $(\mathrm{bpm})$ & $63 \pm 11$ \\
\hline
\end{tabular}


Table 2 - Baseline cardiovascular and hemodynamic parameters

\begin{tabular}{|c|c|c|c|c|c|c|}
\hline & \multicolumn{3}{|c|}{ SED } & \multicolumn{3}{|c|}{ TEA-BREAK } \\
\hline & Pre & Post & $\Delta$ & Pre & Post & $\Delta$ \\
\hline $\mathrm{SAP}(\mathrm{mmHg})$ & $114 \pm 7$ & $118 \pm 8^{*}$ & $3.7 \pm 6.5$ & $114 \pm 7$ & $118 \pm 7^{*}$ & $4.0 \pm 7.4$ \\
\hline DAP (mmHg) & $65 \pm 6$ & $70 \pm 6^{*}$ & $5.5 \pm 5.3$ & $65 \pm 6$ & $71 \pm 8^{*}$ & $5.8 \pm 7.8$ \\
\hline MAP (mmHg) & $81 \pm 5$ & $86 \pm 6^{*}$ & $4.6 \pm 4.6$ & $81 \pm 5$ & $87 \pm 7^{*}$ & $5.2 \pm 6.8$ \\
\hline HR (bpm) & $63 \pm 11$ & $53 \pm 10^{*}$ & $-10 \pm 6$ & $66 \pm 10$ & $56 \pm 9^{*}$ & $-10 \pm 7$ \\
\hline $\operatorname{MCAv}(\mathrm{cm} / \mathrm{s})$ & $68 \pm 9$ & $63 \pm 8^{*}$ & $-5.2 \pm 7.0$ & $62 \pm 12$ & $58 \pm 9^{*}$ & $-4.4 \pm 6.6$ \\
\hline $\mathrm{CVC}_{\mathrm{i}}\left(\mathrm{cm} \cdot \mathrm{s}^{-1} \cdot \mathrm{mmHg}^{-1}\right)$ & $0.85 \pm 0.13$ & $0.74 \pm 0.09^{*}$ & $-0.11 \pm 0.09$ & $0.77 \pm 0.15$ & $0.67 \pm 0.11^{*}$ & $-0.10 \pm 0.10$ \\
\hline $\mathrm{PETCO}_{2}(\mathrm{mmHg})$ & $40 \pm 2$ & $39 \pm 2$ & $-0.62 \pm 1.88$ & $39 \pm 2$ & $37 \pm 2^{*}$ & $-2.2 \pm 1.2^{\#}$ \\
\hline \multicolumn{7}{|c|}{$\begin{array}{l}\text { The results are presented as means } \pm S D . n=19 . \text { Pre- and post-intervention data were analyzed using two-way ANOVA with repeated measurements } \\
\text { followed by Sidak post hoc test. Changes }(\triangle) \text { data were analyzed using paired Student test. * different from PRE; \# different from } S E D ; P<0.05 . C V C_{i^{\prime}} \\
\text { cerebral vascular conductance index; DAP, diastolic arterial pressure; MAP, mean arterial pressure; } M C A v, \text { medium cerebral artery velocity; PETCO }{ }_{2}^{\prime} \\
\text { partial pressure of end-tidal } C O_{2^{\prime}} S A P, \text { systolic arterial pressure; TEA-BREAK, six hours of desk work with walking and tea breaks every hour; SED, } \\
\text { sedentary intervention: six hours of desk work with drinking water (while seated) every hour }\end{array}$} \\
\hline
\end{tabular}

A

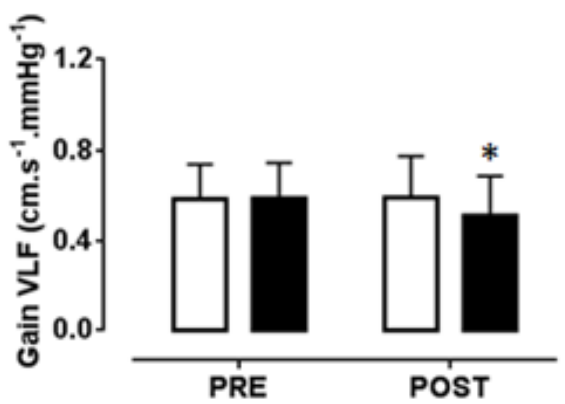

C

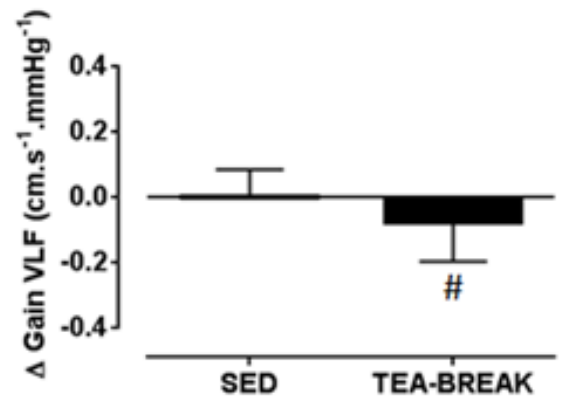

B

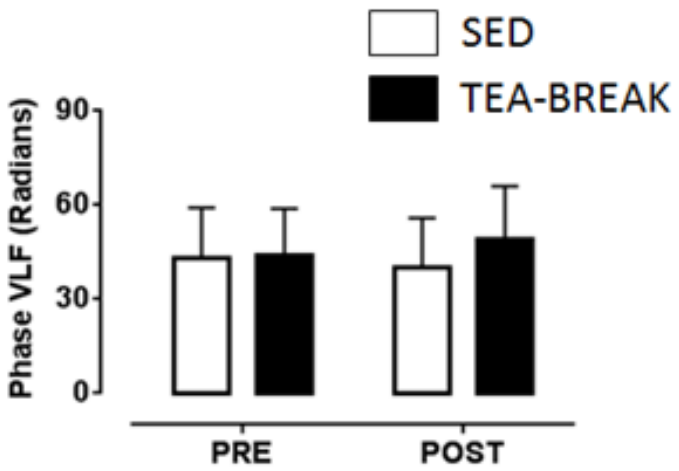

D

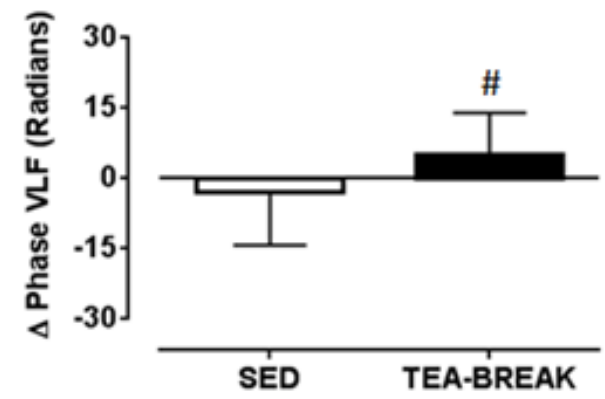

Figure 2 - Cerebral autoregulation in very low frequency (VLF). Absolute values of A) Gain VLF, and B) phase VLF pre and post 6-hour of deskwork with tea breaks every hour (TEA-BREAK) or sedentary intervention drinking water every hour (SED). Changes in C) Gain VLF, and D) Phase VLF in response to 6-hour of deskwork in TEA-BREAK or SED intervention. The results are presented as the means \pm SD. $n=19$. In A and B data were analysed using two-way ANOVA followed by Sidak post hoc test. In D and E data were analysed using paired Student $t$ test. * different from PRE; \# different from SED; $P<0.05$. 
A

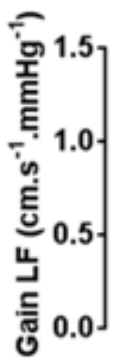

C

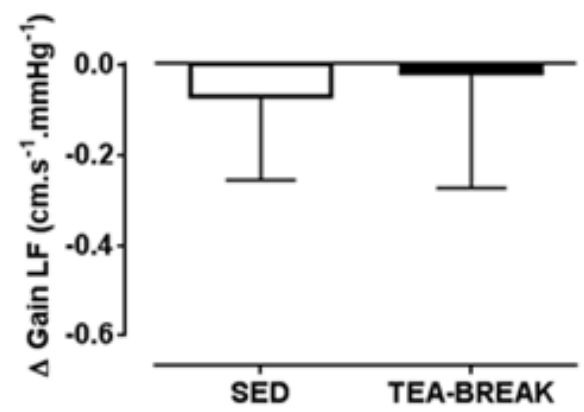

B

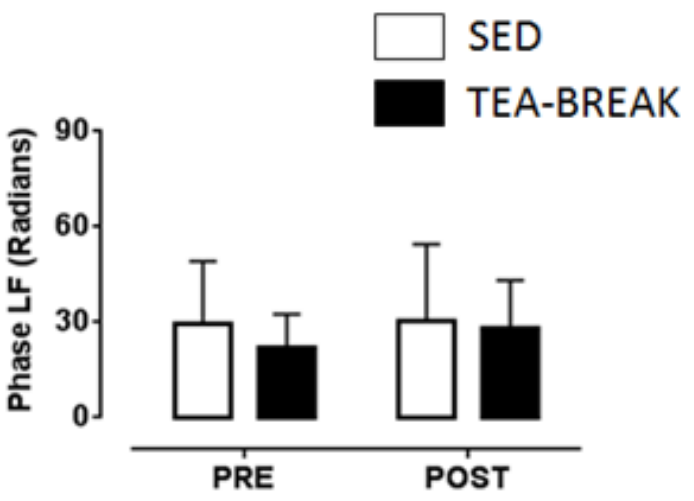

D

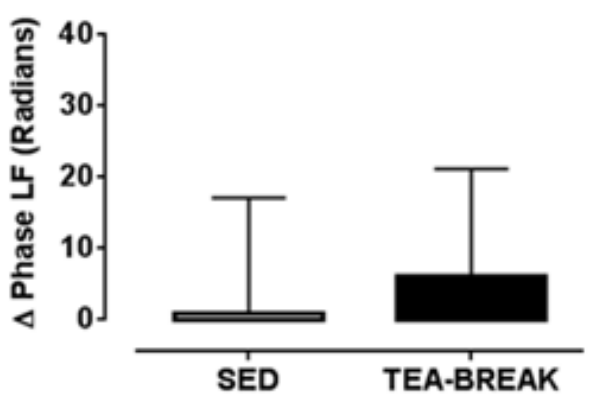

Figure 3 - Cerebral autoregulation in low frequency (LF). Absolute values of A) Gain LF, and B) phase LF pre and post 6-hour of deskwork with tea breaks every hour (TEA-BREAK) or sedentary intervention drinking water every hour (SED). Changes in C) Gain LF, and D) Phase LF in response to 6-hour of deskwork in TEA-BREAK or SED intervention. In A and B data were analysed using two-way ANOVA followed by Sidak post hoc test. In $\mathrm{D}$ and $\mathrm{E}$ data were analysed using paired Student $t$ test. * different from PRE; \# different from SED; $P<0.05$.

more efficient cerebrovascular response to BP changes, while no such effects were found with prolonged sitting. Taken together, these findings confirm the deleterious effects of sedentary work on CBF and affect. Although short breaks with physical activity and tea could not prevent these effects, they significantly improved cerebrovascular function in healthy volunteers.

It is well established that sedentary behavior, which is characterized by little physical movement and low energy expenditure, including prolonged sitting, leads to deleterious health outcomes, including impairment of cardiovascular function. ${ }^{27}$ Indeed, it has been shown that continuous sitting is associated with lower MCAv, but also with lower BP and impaired peripheral vascular function. ${ }^{6,28}$ Our data confirms previous findings in that prolonged desk work lowers blood flow through the middle cerebral artery (MCA). This decline in the CBF may be a consequence of endothelial dysfunction in cerebral blood vessels due to a lower nitric oxide availability as a result of prolonged sitting. ${ }^{29,30}$ Further work should explore potential mechanisms underlying this consistent observation in humans.

In contrast to our hypothesis, breaking up prolonged sitting with light physical activity accompanied by tea consumption did not prevent the decline in MCAv and increase in MAP. This is somewhat surprising, since previous work on the effects of walking breaks during prolonged sitting prevented decreases in MCAv. ${ }^{6} \mathrm{~A}$ possible explanation for the conflicting results is the longer sitting time in our protocol, which could have made it more difficult for short breaks with light physical activity to prevent these decreases. Previous studies demonstrating benefits of physical activity breaks typically adopted more frequent (every $30 \mathrm{~min}$ ) and more intense exercise ${ }^{6,7}$ compared with the present study. Therefore, positive metabolic and cardiovascular 
Table 3 - Mood and affect questionnaires (n=19)

\begin{tabular}{|c|c|c|c|c|c|c|}
\hline & \multicolumn{3}{|c|}{ SED } & \multicolumn{3}{|c|}{ TEA-BREAK } \\
\hline & Pre & Post & $\Delta$ & Pre & Post & $\Delta$ \\
\hline \multicolumn{7}{|l|}{ PANAS } \\
\hline Positive affect & $27 \pm 7$ & $22 \pm 6^{*}$ & $-5.5 \pm 7.3$ & $29 \pm 5$ & $26 \pm 9$ & $-2.9 \pm 7.1$ \\
\hline Negative Affect & $12 \pm 3$ & $11 \pm 2$ & $-1.2 \pm 3.3$ & $13 \pm 4$ & $12 \pm 3$ & $-0.3 \pm 4.0$ \\
\hline \multicolumn{7}{|l|}{ AFFECT GRID } \\
\hline Pleasure-displeasure & $6 \pm 1$ & $6 \pm 1$ & $-0.2 \pm 2.2$ & $6 \pm 1$ & $5 \pm 1$ & $-0.7 \pm 1.6$ \\
\hline Arousal-steepness & $4 \pm 1$ & $4 \pm 2$ & $0.2 \pm 2.8$ & $5 \pm 1$ & $5 \pm 2$ & $0.7 \pm 2.2$ \\
\hline \multicolumn{7}{|l|}{ BOND \& LADER } \\
\hline Alert & $49 \pm 5$ & $50 \pm 7$ & $0.6 \pm 7.6$ & $47 \pm 6$ & $50 \pm 6$ & $3.1 \pm 6.7$ \\
\hline Content & $53 \pm 5$ & $51 \pm 6$ & $-1.5 \pm 5.4$ & $54 \pm 5$ & $56 \pm 6$ & $1.2 \pm 5$ \\
\hline Calm & $54 \pm 13$ & $53 \pm 8$ & $-1.3 \pm 11.1$ & $50 \pm 8$ & $49 \pm 14$ & $-0.8 \pm 16$ \\
\hline \multicolumn{7}{|c|}{$\begin{array}{l}\text { The results are presented as means } \pm \text { SD. Pre-and post-intervention data were analyzed using two-way ANOVA with repeated measurements followed } \\
\text { by Sidak post hoc test. Changes }(\triangle) \text { data were analyzed using paired Student t test. * different from PRE; P<0.05. TEA-BREAK, } 6 \text {-hour of deskwork with } \\
\text { tea breaks every hour; SED, sedentary intervention: six hours of desk work with drinking water (while seated) every hour; PANAS, Positive and Negative } \\
\text { Affect Schedule. }\end{array}$} \\
\hline
\end{tabular}

effects, induced by physical activity, that can improve cerebrovascular function may be intensity- and frequency-dependent. In addition, prolonged sitting seems to increase postprandial glycemia, ${ }^{31}$ resulting in decreases in $\mathrm{MCAv},{ }^{32}$ and frequent physical activity breaks seem to prevent it. ${ }^{31}$ Also, pre-clinical and clinical studies have shown that physical activity causes an increase in CBF by a cholinergic receptor mechanism. ${ }^{33-35}$ Further studies are needed to directly evaluate the effects of different levels of physical activity breaks on cardiovascular and metabolic mechanisms involved in the $\mathrm{CBF}$ regulation.

Additionally, the fact that our experimental design included healthy participants and acute tea ingestion makes it difficult to compare the cardiovascular response with previous studies. The consumption of black tea has been shown to be effective in improving cutaneous vascular function and flow-mediated dilation of the brachial artery. ${ }^{11,12}$ These benefits on peripheral vessels seem to be mediated by tea flavonoids by improving the bioactivity of the endothelium-derived vasodilator nitric oxide. ${ }^{36}$

Nevertheless, there is no conclusive evidence for the impact of tea on cerebral perfusion during prolonged sitting. For instance, black tea flavonoids did not alter
CBF. ${ }^{30}$ At least, data from our study suggests that, in a real-world setting, breaking sedentary work time with low-intensity physical activity combined with tea ingestion does not prevent the impact of prolonged sitting on cerebral perfusion. Importantly, we found a slight reduction in $\mathrm{PETCO}_{2}(-2.2 \mathrm{mmHg})$ after the TEABREAKS, which could promote a reduction in the MCA diameter resulting in changes in the MCAv. Nevertheless, a model based on data from studies that evaluated the influence of changes in MCA diameter on the validity of TCD during changes in $\mathrm{PETCO}_{2}$ showed that substantial changes in MCA diameter occur with changes in $\mathrm{PETCO}_{2}$ $\geq 7.5 \mathrm{mmHg}^{37}$

Although the TEA-BREAKS did not induce changes in MCAv, we found that this intervention improved CA, by reducing gain and increasing phase in response to $\mathrm{BP}$ fluctuations using the $10 \mathrm{~s}$ squatting-standing protocol (VLF). A lower gain denotes a more efficient $C A$, since for a certain change in $\mathrm{BP}$, lower changes in MCAv would be required. ${ }^{22}$ In other words, these data indicate a greater ability of cerebral vessels to prevent large fluctuations in CBF. The higher phase is related to a faster response of cerebral blood vessels to BP variations, which is linked to the efficiency of the system. ${ }^{22}$ These benefits of physical activity breaks combined with tea ingestion 
were not observed when more rapid fluctuations in BP were adopted (i.e. LF). The higher coherence in VLF compared to LF may contribute to this observation. Our findings fit with a previous study showing that walking breaks also enhanced the VLF phase compared with uninterrupted sitting. ${ }^{6}$ In contrast, in a previous work, acute tea ingestion had no effect on CA. ${ }^{38}$ This suggests that physical activity may have a greater impact than tea on CA. However, we could not demonstrate the relative contribution of physical activity or tea in preventing the detrimental effects of prolonged sitting. This represents an important limitation of the present study. Future work is required to better understand these effects.

In the present investigation, prolonged sitting impaired positive affect. Among the different parameters related to mood, we found a decrease in PANAS positive affect after six hours of desk work, but not in PANAS negative affect, Bond \& Lader questionnaire or the Affect grid. The different findings between questionnaires might be attributable to the relatively small number of participants. Nonetheless, the decrease in affect fits with previous work that also linked sedentary behavior to decreases in mood and affect. ${ }^{7}$ In our study, we found no evidence for the ability of waliking breaks combined with tea ingestion to prevent the decline in affect and mood during prolonged sitting. Possibly, the intensity and frequency of walking breaks in our study were not sufficient to enhance affect and mood. ${ }^{7}$

\section{Conclusions}

The results of the present study add to the growing body of literature that prolonged desk work leads to unhealthy outcomes, including an increase in MAP, decreases in MCAv, and lower affect status. Repeated exposure to such physiological disturbances may increase the risk for cardiovascular disease, although future work is required to better understand this link. These effects could not be significantly altered by short walking breaks

\section{References}

1. Owen N, Sparling PB, Healy GN, Dunstan DW, Matthews CE. Sedentary behavior: emerging evidence for a new health risk. Mayo Clin Proc. 2010;85(12):1138-41.

2. WHO. Global recommendations on physical activity for health. Geneva. 2010

3. Krogh-Madsen R, Pedersen M, Solomon TP, Knudsen SH, Hansen LS, Karstoft K, et al. Normal physical activity obliterates the deleterious effects of a high-caloric intake. J Appl Physiol (1985). 2014;116(3):231-9. combined with tea ingestion in healthy individuals. However, tea breaks improved CA, indicating a higher efficiency in maintaining CBF in response to $\mathrm{BP}$ changes. These findings further support the importance of lifestyle changes to prevent the detrimental effects of prolonged, uninterrupted sitting.

\section{Author contributions}

Conception and design of the research: Thijssen DH, Hopkins N, and Low D. Acquisition of data: Speretta GF, Fornasiero A, and John JA. Statistical analysis: Speretta GF. Obtaining financing: Thijssen DH, Hopkins $\mathrm{N}$, and Low DA. Writing of the manuscript: Speretta GF. Critical revision of the manuscript for intellectual content: Thijssen DH, Hopkins N, Low DA, Fornasiero A, and John JA.

\section{Potential Conflict of Interest}

No potential conflict of interest relevant to this article was reported.

\section{Sources of Funding}

This study was funded by Unilever B.V.

\section{Study Association}

This study is not associated with any thesis or dissertation work.

\section{Ethics approval and consent to participate}

This study was approved by the Ethics Committee of the Liverpool John Moores University under the protocol number 19/SPS/023. All the procedures in this study were in accordance with the 1975 Helsinki Declaration, updated in 2013. Informed consent was obtained from all participants included in the study.
4. Pedersen L, Hojman P. Muscle-to-organ cross talk mediated by myokines. Adipocyte. 2012;1(3):164-167.

5. Ekelund U, Ward HA, Norat T, Luan J, May AM, Weiderpass E, et al. Physical activity and all-cause mortality across levels of overall and abdominal adiposity in European men and women: the European Prospective Investigation into Cancer and Nutrition Study (EPIC). Am J Clin Nutr. 2015;101(3):613-21.

6. Carter SE, Draijer R, Holder SM, Brown L, Thijssen DHJ, Hopkins ND. Regular walking breaks prevent the decline in cerebral blood flow associated with prolonged sitting. J Appl Physiol (1985). 2018;125(3):790-8. 
7. Giurgiu M, Koch ED, Plotnikoff RC, Ebner-Priemer UW, Reichert M. Breaking Up Sedentary Behavior Optimally to Enhance Mood. Med Sci Sports Exerc. 2019

8. Duvivier BM, Schaper NC, Hesselink MK, van Kan L, Stienen N, Winkens B, et al. Breaking sitting with light activities vs structured exercise: a randomised crossover study demonstrating benefits for glycaemic control and insulin sensitivity in type 2 diabetes. Diabetologia. 2017;60(3):490-8.

9. Einother SJ, Martens VE. Acute effects of tea consumption on attention and mood. Am J Clin Nutr. 2013;98(6 Suppl):1700S-1708S.

10. Tijburg LB, Mattern T, Folts JD, Weisgerber UM, Katan MB. Tea flavonoids and cardiovascular disease: a review. Crit Rev Food Sci Nutr. 1997;37(8):771-85.

11. Schreuder TH, Eijsvogels TM, Greyling A, Draijer R, Hopman MT, Thijssen DH. Effect of black tea consumption on brachial artery flowmediated dilation and ischaemia-reperfusion in humans. Appl Physiol Nutr Metab. 2014;39(2):145-51.

12. Woodward KA, Hopkins ND, Draijer R, de Graaf Y, Low DA, Thijssen DHJ. Acute black tea consumption improves cutaneous vascular function in healthy middle-aged humans. Clin Nutr. 2018;37(1):242-9.

13. Arab L, Liu W, Elashoff D. Green and black tea consumption and risk of stroke: a meta-analysis. Stroke. 2009;40(5):1786-92.

14. Thijssen DH, Black MA, Pyke KE, Padilla J, Atkinson G, Harris RA, et al. Assessment of flow-mediated dilation in humans: a methodological and physiological guideline. Am J Physiol Heart Circ Physiol. 2011;300(1):H2-12.

15. van Mil AC, Hartman Y, van Oorschot F, Heemels A, Bax N, Dawson EA, et al. Correlation of carotid artery reactivity with cardiovascular risk factors and coronary artery vasodilator responses in asymptomatic, healthy volunteers. J Hypertens. 2017;35(5):1026-34.

16. Morishima T, Restaino RM, Walsh LK, Kanaley JA, Fadel PJ, Padilla J. Prolonged sitting-induced leg endothelial dysfunction is prevented by fidgeting. Am J Physiol Heart Circ Physiol. 2016;311(1):H177-82.

17. Skirton H, Chamberlain W, Lawson C, Ryan H, Young E. A systematic review of variability and reliability of manual and automated blood pressure readings. J Clin Nurs. 2011;20(5-6):602-14.

18. Waldron M, David Patterson S, Jeffries O. Inter-Day Reliability of Finapres. Sports Med Int Open. 2018;2(1):E9-E15.

19. Willie CK, Colino FL, Bailey DM, Tzeng YC, Binsted G, Jones LW, et al. Utility of transcranial Doppler ultrasound for the integrative assessment of cerebrovascular function. J Neurosci Methods. 2011;196(2):221-37.

20. Skow RJ, MacKay CM, Tymko MM, Willie CK, Smith KJ, Ainslie PN, et al. Differential cerebrovascular $\mathrm{CO}(2)$ reactivity in anterior and posterior cerebral circulations. Respir Physiol Neurobiol. 2013;189(1):76-86.

21. Smirl JD, Hoffman K, Tzeng YC, Hansen A, Ainslie PN. Methodological comparison of active- and passive-driven oscillations in blood pressure; implications for the assessment of cerebral pressure-flow relationships. J Appl Physiol (1985). 2015;119(5):487-501.

22. van Beek AH, Claassen JA, Rikkert MG, Jansen RW. Cerebral autoregulation: an overview of current concepts and methodology with special focus on the elderly. J Cereb Blood Flow Metab. 2008;28(6):1071-85.

23. Claassen JA, Meel-van den Abeelen AS, Simpson DM, Panerai RB, international Cerebral Autoregulation Research N. Transfer function analysis of dynamic cerebral autoregulation: A white paper from the International Cerebral Autoregulation Research Network. J Cereb Blood Flow Metab. 2016;36(4):665-80.

24. Watson D, Clark LA, Tellegen A. Development and validation of brief measures of positive and negative affect: the PANAS scales. J Pers Soc Psychol. 1988;54(6):1063-70.

25. Bond A, Lader $\mathrm{M}$. The use of analogue scales in rating subjective feelings. Br J Psychol. 1974;47:211-8.

26. Russell YI, Gobet F. Sinuosity and the Affect Grid: a method for adjusting repeated mood scores. Percept Mot Skills. 2012;114(1):125-36.

27. Owen N, Healy GN, Matthews CE, Dunstan DW. Too much sitting: the population health science of sedentary behavior. Exerc Sport Sci Rev. 2010;38(3):105-13.

28. Wheeler MJ, Dunstan DW, Smith B, Smith KJ, Scheer A, Lewis J, et al. Morning exercise mitigates the impact of prolonged sitting on cerebral blood flow in older adults. J Appl Physiol (1985). 2019;126(4):1049-55.

29. Sabayan B, Westendorp RG, Grond J, Stott DJ, Sattar N, van Osch MJ, et al. Markers of endothelial dysfunction and cerebral blood flow in older adults. Neurobiol Aging. 2014;35(2):373-7.

30. Toda N. Age-related changes in endothelial function and blood flow regulation. Pharmacol Ther. 2012;133(2):159-76.

31. Dunstan DW, Kingwell BA, Larsen R, Healy GN, Cerin E, Hamilton MT, et al. Breaking up prolonged sitting reduces postprandial glucose and insulin responses. Diabetes Care. 2012;35(5):976-83.

32. Wheeler MJ, Dempsey PC, Grace MS, Ellis KA, Gardiner PA, Green DJ, et al. Sedentary behavior as a risk factor for cognitive decline? A focus on the influence of glycemic control in brain health. Alzheimers Dement (N Y). 2017;3(3):291-300.

33. Seifert T, Fisher JP, Young CN, Hartwich D, Ogoh S, Raven PB, et al Glycopyrrolate abolishes the exercise-induced increase in cerebral perfusion in humans. Exp Physiol. 2010;95(10):1016-25.

34. Seifert T, Secher NH. Sympathetic influence on cerebral blood flow and metabolism during exercise in humans. Prog Neurobiol. 2011;95(3):406-26

35. Toda N, Ayajiki K, Tanaka T, Okamura T. Preganglionic and postganglionic neurons responsible for cerebral vasodilation mediated by nitric oxide in anesthetized dogs. J Cereb Blood Flow Metab. 2000;20(4):700-8.

36. Ras RT, Zock PL, Draijer R. Tea consumption enhances endothelialdependent vasodilation; a meta-analysis. PLoS One. 2011;6(3):e16974.

37. Hoiland RL, Fisher JA, Ainslie PN. Regulation of the Cerebral Circulation by Arterial Carbon Dioxide. Compr Physiol. 2019;9(3):1101-54.

38. Vidyasagar R, Greyling A, Draijer R, Corfield DR, Parkes, LM. The effect of black tea and caffeine on regional cerebral blood flow measured with arterial spin labeling. Journal of Cerebral Blood Flow \& Metabolism. 2013;33:963-968. 